\title{
A Series Expansion for the First Positive Zero of the Bessel Functions
}

\section{By R. Piessens}

$$
\begin{aligned}
& \text { Abstract. It is shown that the first positive zero } j_{\nu, 1} \text { of the Bessel function } J_{\nu}(x) \text { is given by } \\
& \qquad J_{v \cdot 1}=2(\nu+1)^{1 / 2}\left[1+\frac{(\nu+1)}{4}-\frac{7(\nu+1)^{2}}{96}+\frac{49(\nu+1)^{3}}{1152}-\frac{8363(\nu+1)^{4}}{276480}+\cdots\right] \\
& \text { for }-1<\nu<0 \text {. }
\end{aligned}
$$

1. It is well known that, when $\nu$ is real and $\nu>-1$, the Bessel function $J_{\nu}(x)$ has an infinite number of zeros and that all zeros are real (Watson [9]). We denote the $s$ th positive zero of $J_{\nu}(x)$ by $j_{\nu, s}$.

Several approximations, asymptotic expansions or bounds for the zeros of Bessel functions exist (see [1], [2], [4], [6], [7], [9]). Especially McMahon's expansion for large zeros (see Abramowitz and Stegun [1]), Olver's asymptotic expansion for large orders and Olver's uniform asymptotic expansions (see Olver [6]) are interesting formulas, but, unfortunately, they are not applicable when $s$ and $\nu$ are small. The purpose of this note is to give a series expansion for $j_{\nu, 1}$ when $-1<\nu<0$.

2. Cayley [3] noticed that Graeffe's method for solving a polynomial equation can be applied for the efficient computation of

$$
\sum_{s=1}^{\infty} j_{\nu, s}^{-2 r} \equiv \sigma_{\nu}^{(r)}, \quad r=1,2, \ldots .
$$

An upper bound for $j_{\nu, 1}$ is given by Chambers [4]:

$$
j_{\nu, 1}<(\nu+1)^{1 / 2}\left[(\nu+2)^{1 / 2}+1\right] \text {. }
$$

Further it is known that, when $k>1$,

$$
\lim _{\nu \rightarrow-1} j_{\nu, k}=j_{1, k-1}>0 \text {. }
$$

Thus, the first term in the left side of (1) is dominant when $\nu \simeq-1$, so that

$$
j_{\nu, 1}=2(\nu+1)^{1 / 2} \phi_{r}(\nu)+o\left((\nu+1)^{r-1}\right), \quad \nu \rightarrow-1,
$$

where

$$
\phi_{r}(\nu)=\left[\frac{1}{2^{2 r}(\nu+1)^{r} \sigma_{\nu}^{(r)}}\right]^{1 / 2 r}
$$

is analytic at $\nu=-1$.

Received November 22, 1982.

1980 Mathematics Subject Classification. Primary 33A40, 49G10. 
By approximating $\phi_{r}(\nu)$ by a Taylor polynomial, we obtain

$$
j_{\nu, 1}=2(\nu+1)^{1 / 2} \sum_{k=0}^{r-1} C_{k}(\nu+1)^{k}+o\left((\nu+1)^{r-1}\right), \quad \nu \rightarrow-1,
$$

where

$$
C_{k}=\left.\frac{1}{k !} \frac{d^{k} \phi_{r}(\nu)}{d \nu^{k}}\right|_{\nu=-1}
$$

is independent of $r$.

When $r \rightarrow \infty$, (6) becomes a series expansion for $j_{\nu, 1}$, which, because of the presence of a branchpoint of $\phi_{r}(\nu)$ at $\nu=-2$, converges only in the interval $-1<\nu<0$.

Using REDUCE, which is a computer language for formula manipulation [5], we have computed $C_{k}, k=0,1,2,3,4$, using (7), where $r=8$ and

(8) $\phi_{8}(\nu)=\left[\frac{(\nu+2)^{4}(\nu+3)^{2}(\nu+4)^{2}(\nu+5)(\nu+6)(\nu+7)(\nu+8)}{429 \nu^{5}+7640 \nu^{4}+53752 \nu^{3}+185430 \nu^{2}+311387 \nu+202738}\right]^{1 / 16}$.

The result is

(9)

$j_{\nu, 1}=2(\nu+1)^{1 / 2}\left[1+\frac{(\nu+1)}{4}-\frac{7(\nu+1)^{2}}{96}+\frac{49(\nu+1)^{3}}{1152}-\frac{8363(\nu+1)^{4}}{276480}+\ldots\right]$.

In Table 1, we compare the exact values of $j_{\nu, 1}$ with the approximate values given by (9), for $\nu=-3 / 4,-2 / 3,-1 / 2,-1 / 3,-1 / 4$ and also for $\nu=0$ (although we were not able to prove the convergences of the expansion for $\nu=0$ ).

\section{TABLE 1}

Values of the first zero $j_{\nu, 1}$ of $J_{\nu}(x)$

\begin{tabular}{|rcc|}
\hline \multicolumn{1}{|c}{$\nu$} & exact & approximation (9) \\
\hline$-3 / 4$ & 1.058508 & 1.058489 \\
$-2 / 3$ & 1.243046 & 1.242958 \\
$-1 / 2$ & 1.570796 & 1.570056 \\
$-1 / 3$ & 1.866351 & 1.863061 \\
$-1 / 4$ & 2.006300 & 2.000273 \\
0 & 2.404826 & 2.378740 \\
\hline
\end{tabular}

3. An interesting application of (9) is the estimation of the smallest zero of Laguerre- and Gegenbauer-polynomials [8]. For example, the smallest zero $\xi_{n}$ of the Laguerre polynomials $L_{n}^{(\alpha)}(x)$ is approximated by (see Tricomi [8])

$$
\xi_{n}=\frac{j_{\alpha, 1}}{4 k_{n}}\left[1+\frac{2\left(\alpha^{2}-1\right)+j_{\alpha, 1}^{2}}{48 k_{n}^{2}}\right]
$$

where $k_{n}=n+(\alpha+1) / 2$. In Table 2, this approximation, where $j_{\alpha, 1}$ is replaced by (9), is compared with the exact value of $\xi_{n}$. 
TABLE 2

Values of the smallest zero of $L_{n}^{(\alpha)}(x)$

\begin{tabular}{|crcc|}
\hline \multicolumn{1}{c}{$\boldsymbol{n}$} & $\boldsymbol{n}$ & exact & approximation (10) \\
\hline$-3 / 4$ & 3 & 0.089682 & 0.089679 \\
& 15 & 0.018520 & 0.018519 \\
\hline$-1 / 2$ & 3 & 0.190163 & 0.189982 \\
& 15 & 0.040452 & 0.040415 \\
\hline$-1 / 4$ & 3 & 0.299347 & 0.297530 \\
& 15 & 0.065463 & 0.065071 \\
\hline 0 & 3 & 0.415775 & 0.406686 \\
& 15 & 0.093308 & 0.091294 \\
\hline
\end{tabular}

Acknowledgement. This research is supported by the 'Nationaal Fonds voor Wetenschappelijk Onderzoek (Belgium)'.

Department of Computer Science

University of Leuven

Celestijnenlaan $200 \mathrm{~A}$

B-3030 Heverlee, Belgium

1. M. Abramowitz \& I. Stegun, Handbook of Mathematical Functions, Dover, New York, 1965.

2. M. Branders, R. Piessens \& M. De MeUe, "Rational approximations for zeros of Bessel functions," J. Comput. Phys., v. 42, 1981, pp. 403-405.

3. A. CaYley, "Addition to Lord Rayleigh's paper: On the numerical calculation of the roots of fluctuating functions," Proc. London Math. Soc., v. 5, 1873-1874, pp. 123-124.

4. L. G. Chambers, “An upper bound for the first zero of Bessel functions,” Math. Comp., v. 38, 1982, pp. 589-591.

5. A. C. HeARn, REDUCE 2-A System and Language for Algebraic Manipulation, Proc. Second Sympos. on Symbolic and Algebraic Manipulation, Los Angeles, 1971, pp. 128-133.

6. F. W. J. Olver, Royal Society Mathematical Tables, Vol. 7, Bessel Functions. Part III. Zeros and Associated Values, The University Press, Cambridge, 1960.

7. N. M. TEMME, "An algorithm with ALGOL 60 program for the computation of the zeros of ordinary Bessel functions and those of their derivatives," J. Comput. Phys., v. 32, 1979, pp. 270-279.

8. F. G. Tricomi, Vorlesungen über Orthogonalreihen, Springer-Verlag, Berlin, 1955.

9. G. N. Watson, A Treatise on the Theory of Bessel Functions, The University Press, Cambridge, 1944. 